\title{
De la bóveda de medio punto a la bóveda escarzana en los puentes de piedra. Influencia del rebajamiento y del relleno rígido en la variación de la carga de rotura
}

\author{
From the semicircular vault to the flattened vault in masonry bridges. The \\ influence of rise/span ratio and the resistant backfil in the breaking load
}

\author{
J. R. Urruchi-Rojo $^{(*)}$, J. A. Martínez-Martínez ${ }^{(*)}$, R. Serrano-López ${ }^{(*)}$
}

\section{RESUMEN}

La forma de las bóvedas de los puentes de piedra ha ido evolucionando con el tiempo, existiendo bibliografía en la que además de recomendarse una tipología frente a otra, según la época, se prescribía la manera de ejecutar el relleno del trasdós. Todo ello surgía en un principio como fruto de las experiencias adquiridas durante la construcción y observación de los puentes. Así fueron emergiendo, a partir del siglo xviI, formulaciones que en unos casos aportaban un resultado y en otros el contrario. Este artículo pretende realizar una comparación entre varias de estas formulaciones, así como efectuar un análisis de la influencia en la variación de la carga de rotura, tanto del rebajamiento de la bóveda como de la participación del relleno rígido en el trasdós del mismo.

Palabras clave: puentes de piedra; arco; rebajamiento; relleno rígido; bóveda.

\section{ABSTRACT}

The shape of the vaults in masonry bridges has evolved during the history, so that we can find bibliography where, besides recommending one way of build instead of others depending on the period, is also prescribed the way to carry out the backfill of the extrados. All of this emerged initially as a result of the experiences acquired during the construction and the observation of bridges. Hence, during the 18th century new formulations started to arise, but they led to one result in some cases and to the opposite in others. This article tries to make a comparison between some of these formulations, as well as carrying out an analysis of the influence of both, the lowering rise/span ratio of the vaults and the presence of a resistant backfill in its extrados, on the variation of the breaking load.

Keywords: masonry bridges; arch; rise/span ratio; resistant backfill; vault.

(*) Departamento de Ingeniería Civil, Universidad de Burgos (Burgos, España).

Persona de contacto/Corresponding author: jrurruchi@gmail.com (J. R. Urruchi-Rojo)

ORCID: http://orcid.org/oooo-ooo1-5201-5229 (J. R. Urruchi-Rojo); http://orcid.org/oooo-ooo3-2153-3183

(J. A. Martínez-Martínez); http://orcid.org/oooo-00o2-7156-4693 (R. Serrano-López)

Cómo citar este artículo/Citation: Urruchi-Rojo, J. R., Martínez-Martínez, J. A., Serrano-López, R. (2016). De la bóveda de medio punto a la bóveda escarzana en los puentes de piedra. Influencia del rebajamiento y del relleno rígido en la variación de la carga de rotura. Informes de la Construcción, 69(545): e187, doi: http://dx.doi.org/10.3989/ic.15.107.

Copyright: (C) 2017 CSIC. Licencia / License: Salvo indicación contraria, todos los contenidos de la edición electrónica de Informes de la Construcción se distribuyen bajo una licencia de uso y distribución Creative Commons Attribution License (CC BY) Spain 3.o. 


\section{INTRODUCCIÓN}

Habría que remontarse más de 4.000 años y trasladarse a Mesopotamia para buscar el origen de las bóvedas de piedra en puentes, correspondiendo en aquel caso a bóvedas de ladrillo secados al sol. En la construcción de puentes de piedra el arco es uno de los elementos fundamentales, ya contemplado así en textos clásicos como el de Alberti en su Tratado de re aedificatoria, el de Vitruvio, en Los diez libros de arquitectura (S. I a.c.), o el de Andrea Palladio (Venecia, 1570) en Los cuatro libros de arquitectura, y más recientemente recordado por un sinfín de autores entre los que cabe citar a Huerta Fernández (1) , Arenas de Pablo (2) o Fernández Casado, C. (3).

Por ello ha sido objeto de innumerables estudios, observaciones y análisis a lo largo de la Historia. Se han tenido en cuenta variables como su forma, el espesor de su bóveda, la luz, la flecha y sus relaciones entre sí. Todo ello con objeto de conocer, en unos casos, los motivos por los que ha predominado un tipo sobre el resto y las reglas que se seguían en cada época, y, en otros casos, para obtener información sobre su comportamiento estructural óptimo y sobre el grado de conocimientos que se tenía en la antigüedad sobre estos aspectos. En cualquier caso debe de tenerse en cuenta que el uso de unas u otras formas, en ocasiones, obedecía a la utilización de prácticas constructivas basadas en la tradición y a la copia de tipologías utilizadas en lugares geográficamente próximos, lo que a veces traía como consecuencia la adopción de hipótesis no bien contrastadas.

En algunos trabajos se ha estudiado la relación entre forma y estructura, comprobando la influencia de la eficiencia y la adaptación estructural en la propia evolución de algunas tipologías a lo largo de la Historia de la Construcción (4). Para el caso particular de los puentes de piedra esa evolución se observa en los cambios geométricos de la bóveda, de manera que su radio de curvatura ha ido aumentando con el paso del tiempo, pudiéndose hablar de un cambio evolutivo similar al de las especies, lo que a Bauder (5) le serviría de argumento para consolidar su teoría sobre ese carácter de seres humanos, que atribuye a los puentes de fábrica. Esa tendencia hacia formas cada vez más rebajadas, en busca de tipos más estilizados y estéticos, ha llevado aparejada variaciones en el espesor de la bóveda a fin de mantener su estabilidad y resis- tencia, lo cual también ha redundado sobre el aspecto exterior de los mismos.

A nivel nacional las actuaciones sobre las bóvedas de los puentes de piedra ha sido estudiada de forma intensa por el grupo de trabajo especializado de la Asociación Técnica de Carreteras (ATC-AIPCR), tanto desde un punto de vista constructivo como estructural; en sus publicaciones se analizan las bases para su análisis, sus mecanismos de trabajo interno, así como posibles modelos a utilizar para su comprobación (6), (7), (8).

Desde un punto de vista resistente, la influencia del rebajamiento en la carga de rotura de puentes en bóveda de piedra fue recientemente analizada por González Parejo (9) , realizando el estudio estructural sobre una serie de geometrías en las que el ángulo del arco variaba entre $180^{\circ}$ y $130^{\circ}$, lo cual equivale a unas relaciones flecha/luz comprendidas entre $1 / 2$ y $1 / 3,14$, respectivamente. En este caso pretendemos analizar también desde un punto de vista estructural la geometría de la bóveda de directriz circular única, completando el rango analizado por González Parejo hasta un rebajamiento de 1/20, así como la influencia del relleno rígido en el trasdós en dicho comportamiento, habida cuenta de su presencia más o menos generalizada en muchos de los puentes sobre los que hemos actuado a lo largo de nuestra carrera profesional. Este último aspecto fue ya puesto de manifiesto en el trabajo de clasificación efectuado por Ramos Casquero y León González (10) sobre bóvedas de fábrica.

\section{ANÁLISIS GEOMÉTRICO}

El parámetro que permite distinguir entre un tipo de arco de directriz circular única u otro es el rebajamiento, que proporciona la relación entre la flecha y la luz. Para determinar numéricamente el valor del mismo es preciso analizar la geometría del arco, que puede estudiarse en función de los parámetros básicos indicados en la Figura 1, en la cual se han señalado además sus partes y los elementos constructivos más importantes siguiendo la terminología comúnmente aceptada para éstos.

Llamando $\mu$ a la relación entre $f$ y $L$ (en adelante referido como rebajamiento), se pueden deducir las siguientes expresiones:

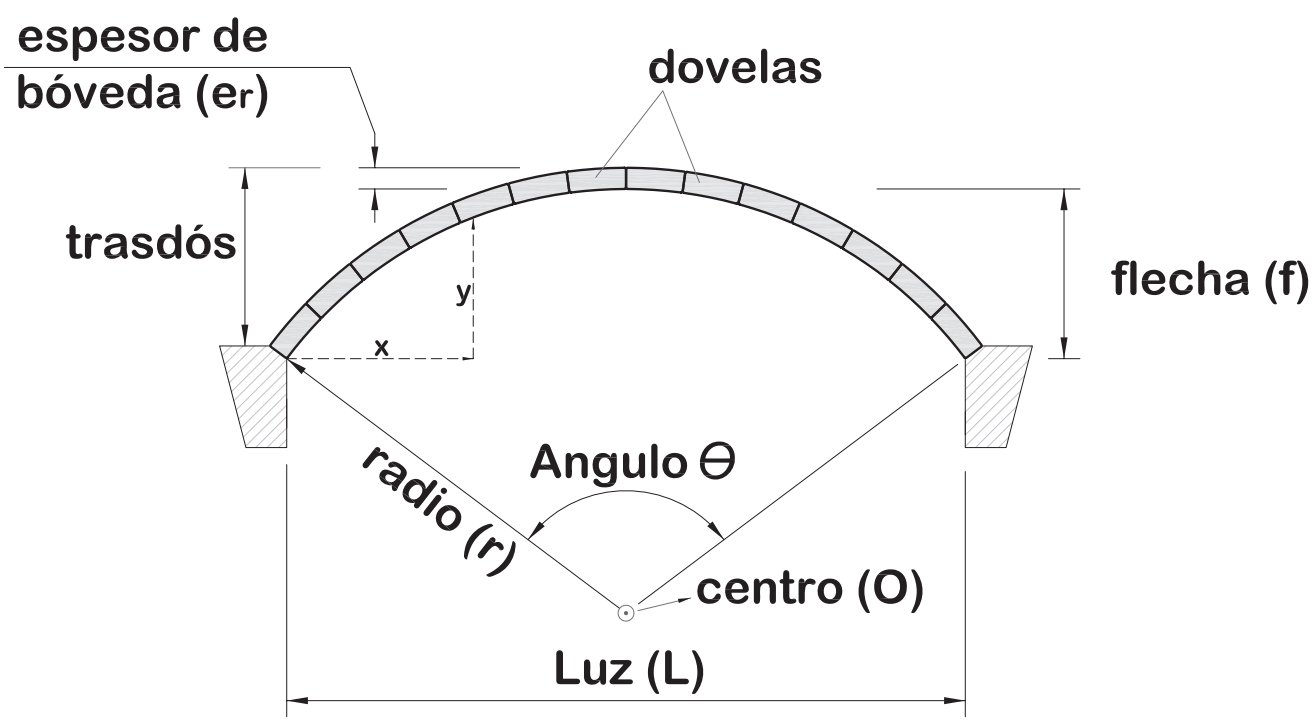

Figura 1. Esquema de un arco de fábrica con sus elementos principales y parámetros geométricos básicos. El conjunto de dovelas constituyen la bóveda. 

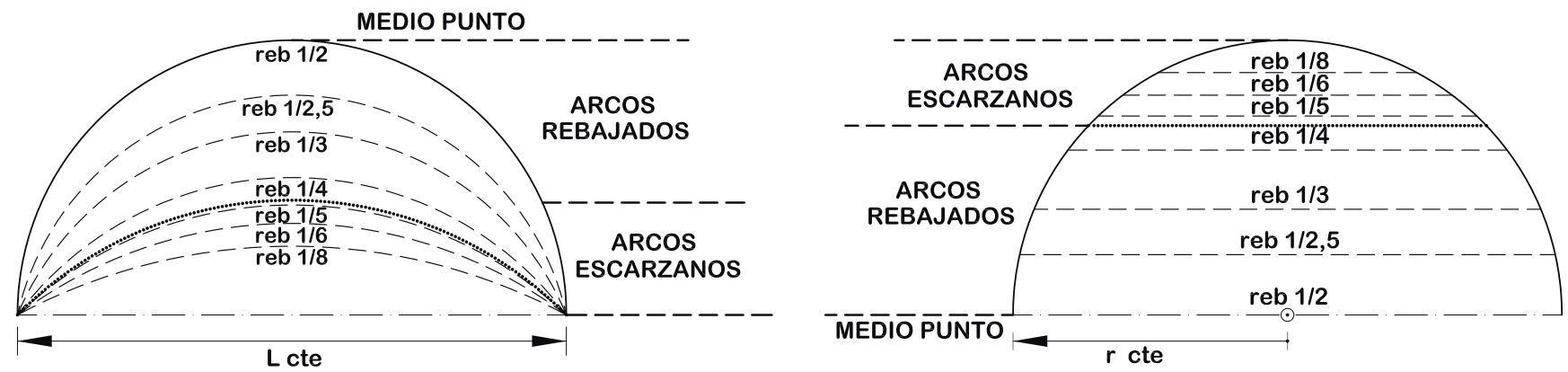

Figura 2. Tipos de arcos de directriz circular única en función del rebajamiento, para luz o radio constantes.

$$
\begin{gathered}
r=\frac{1+4 \mu^{2}}{8 \mu} L \\
\theta=4\left(\frac{\pi}{2}-\operatorname{arctg}\left(\frac{0,5}{\mu}\right)\right)
\end{gathered}
$$

Obteniendo así el ángulo para diferentes rebajamientos del arco.

Atendiendo a las expresiones anteriores y tomando como punto de partida las definiciones contenidas en el glosario de términos empleados en puentes de fábrica (11), Manjón (12) incluyó en su Tesis sobre puentes de fábrica del río Arlanza la siguiente clasificación para tres tipos de arcos de directriz circular única en función del rebajamiento (Figura 2), pudiendo observar un ejemplo de cada uno de ellos (Figura 3):

- Arco de medio punto. El arco de medio punto está constituido por un sector de circunferencia de $180^{\circ}$, fácil de replantear. Entre sus ventajas se encuentra la facilidad de realizar su montaje, ya que todas sus dovelas son iguales. Su rebajamiento es $1 / 2$.

- Arco circular rebajado. Segmento de arco de un único radio, de desarrollo inferior de $180^{\circ}$ y superior a $90^{\circ}$, y que en el caso extremo coincidiría con el arco de medio punto. $\mathrm{Su}$ centro queda por debajo de la línea de los arranques. Su rebajamiento está comprendido entre $1 / 2$ y 1/4,82.

- Arco escarzano. Segmento de arco de un único radio, de desarrollo $\theta$ inferior a $90^{\circ}$. Su centro queda por debajo de la línea de los arranques. El rebajamiento a partir del cual los arcos serían escarzanos viene dado por la siguiente expresión: $\mu \leq \frac{1}{4,82}$

Se puede apreciar (Figura 2) que es posible obtener un determinado rebajamiento, manteniendo la luz, variando el radio (un aumento del radio provoca aumento del rebajamiento, para igual luz), o bien manteniendo el radio y variando la luz (reduciendo la luz aumenta el rebajamiento, para igual radio). Esta segunda opción, manteniendo el radio de curvatura, tiene la ventaja de mecanizar los sillares de los arcos, simplificando el proceso de ejecución e independizándolo de la luz de la bóveda siempre que las pilas puedan situarse a una distancia similar. Por ello, solamente resulta práctica de aplicar hasta un rebajamiento que no reduzca la luz en exceso, como pudiera ser el rebajamiento de $1 / 3$, que implica una reducción de la luz del $8 \%$; pues un rebajamiento de $1 / 4$ ya supone una reducción de luz del $20 \%$.

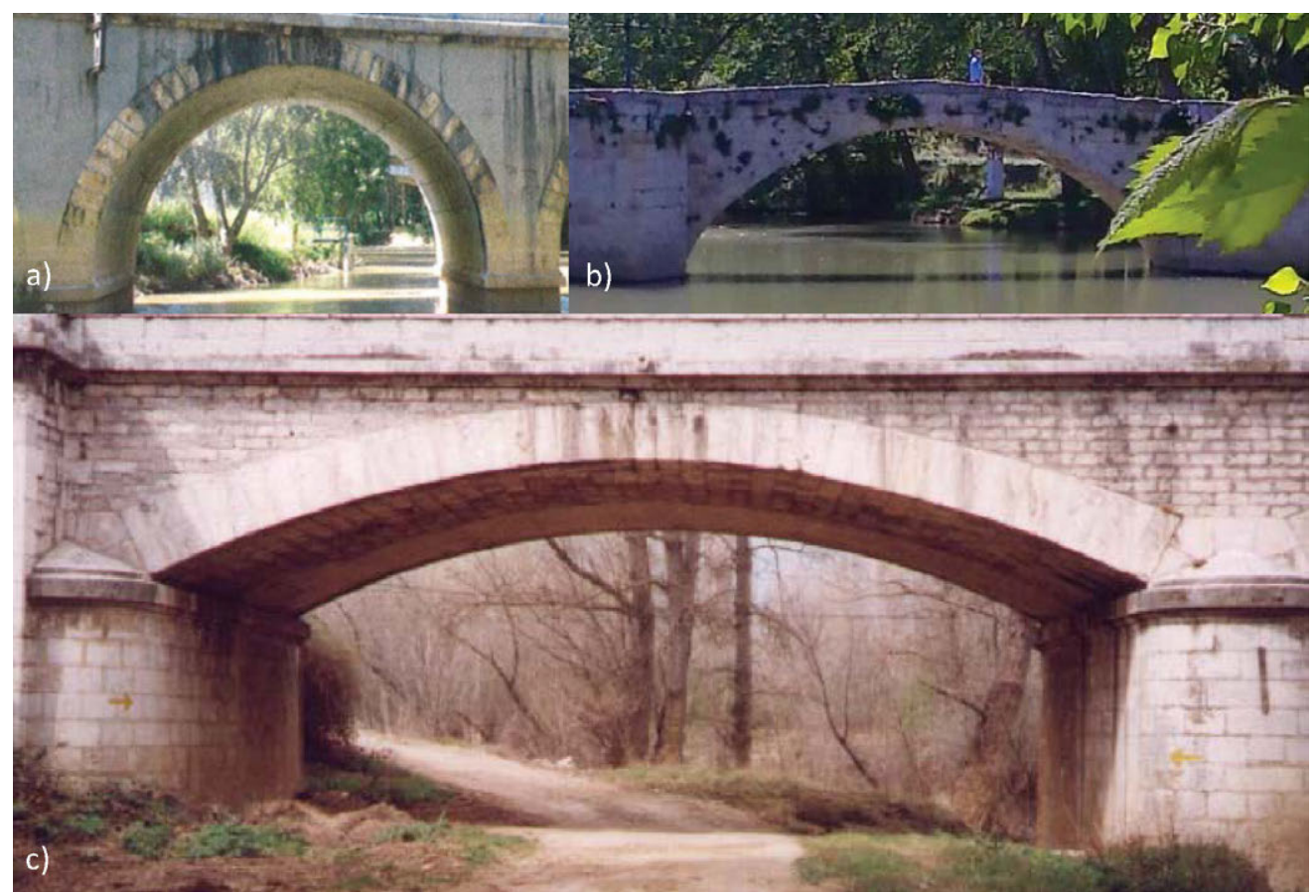

Figura 3. Puentes con diferente directriz circular en la cuenca del río Carrión (fotografías de los autores): a) Puente del Ferrocarril en Dueñas, con bóvedas de medio punto; $b$ ) Puente de Puentecillas en Palencia, con bóvedas rebajadas; c) Puente de San Isidro en Dueñas, con bóveda escarzana. 


\section{EL REBAJAMIENTO DE LA BÓVEDA EN LA BIBLIOGRAFÍA ARQUITECTÓNICA}

En la construcción de bóvedas en puentes se ha aprendido sobre todo de los puentes romanos, tanto para reproducir las virtudes como para rectificar los defectos. Ello ha completado la experiencia constructiva acumulada por la tradición. Los constructores de puentes medievales fueron los primeros en contar con esa gran herencia, pero han sido escasos los ejemplos de bóvedas rebajadas que han llegado hasta nosotros, habiendo predominado la bóveda de medio punto. Conocemos algún ejemplo con ese tipo de bóveda, como el puente de Alconétar (Cáceres), citado por González Parejo (9), que le describe de forma clara, como un exponente de puente romano con bóveda rebajada. De las bóvedas de medio punto de esa época se han llegado a obtener relaciones entre el espesor de la bóveda y la luz, sin que se haya podido deducir ninguna diferenciación en función de su rebajamiento.

Huerta Fernández (1) efectúa una detallada evolución de las técnicas constructivas de arcos, indicando que la caída del Imperio Romano produjo un retroceso en ese aspecto, y que las reglas de geometría práctica aplicadas por romanos y bizantinos, procedentes de la geometría griega, se fueron abandonando. Igualmente indica que durante el siglo xvı, en numerosos tratados que hacen alusión a obras del gótico, se menciona la utilización ya en ese momento de reglas geométricas, que indicaban que las pilas y estribos para arcos rebajados debían ser más gruesos que para arcos de medio punto, y mayor para éstos que para arcos apuntados, pero sin entrar en más detalles. También pone de manifiesto que Martínez de Aranda en el siglo xvi estableció, en un manuscrito sobre cantería, relaciones entre los espesores de las dovelas y la luz de los arcos: $1 / 6,1 / 8$ y 1/10 para luces de 5-10 pies, 10-20 pies y 20-40 pies, respectivamente.

El Tratado re Aedificatoria de Alberti, tal y como describen Aramburu-Zabala (13) y Huerta Fernández (1) entre otros, distingue en los puentes cuatro partes: el muro de contención en las riberas, las pilas, las bóvedas y la calzada. En lo concerniente a los arcos recomendaba que fuesen de medio punto indicando que si se rebajasen se tendría que tener en cuenta en los apoyos de la orilla; que el espesor de los arcos fuese la décima parte del diámetro, y la longitud de éste fuese entre 4 y 6 veces el espesor de las pilas, en cuanto al relleno de arcos recomendaba que se hiciera con piedras sin defectos.

La influencia del Tratado de Alberti en los arquitectos españoles fue importante como refleja F. Marías y Bustamante (14), cuando habla del tratado anónimo español, en el cual se adoptaban similares recomendaciones. No se puede perder de vista que la utilización de unas u otras formas, en ocasiones, obedecía a la utilización de prácticas constructivas basadas en la tradición y a la copia de tipologías utilizadas en lugares geográficamente próximos, lo que a veces traía como consecuencia la utilización de hipótesis no bien contrastadas. Hecho este inciso, citar entre los Tratados españoles que dedican comentarios a los puentes de mediados del siglo xvı, como también recuerda Aramburu-Zabala (13), «Los 21 libros de los ingenios y de las máquinas de Juanelo», del aragonés Pedro Juan de Lastanosa, seguidor de Alberti. Lastanosa da todo un sistema de proporciones muy detallado, no descartando el arco rebajado, aunque para éste establece para las dovelas un espesor de la doceava parte de la luz del arco. Esto viene a indicar que en esa época para el arco de medio punto se estarían utilizando espesores menores y por tanto relaciones entre el espesor del arco y la luz menores de 1/12, como la relación atribuida por Martín-Caro (15) para ese parámetro a Alberti $\left(e_{r} / L=1 / 15\right)$.

Otra influencia clara posterior, ya en el siglo xviı, fue la de Fray Lorenzo de San Nicolás, el cual se deja llevar por la gran cantidad de puentes arruinados en esa época y se convierte en un teórico conservador que entre otros aspectos, y atendiendo exclusivamente a lo relacionado con los arcos, recomienda el de medio punto y la colocación de relleno compactado en el trasdós de las bóvedas, recomendando macizar las pilas hasta los dos tercios de los arcos, como destaca Huerta Fernández (1), (16). Este valor es el utilizado en sus análisis por González Parejo (9), y para el que indica Ramos Casquero (17), que es la altura de relleno que consigue la configuración más estable para la bóveda cañón de directriz circular.

Durante el siglo xviII predominó el cálculo analítico, basado en líneas de presión, por lo que, aplicando esos nuevos conceptos, los diferentes ingenieros de la época fueron formulando expresiones apoyadas en la observación de estructuras existentes y en su propia experiencia, que fueron utilizadas para dimensionar un gran número de estructuras. Estas formulaciones tienen diferentes niveles de sofisticación en las propuestas, desde aquellas donde el canto en clave de la bóveda depende exclusivamente de la luz libre del arco, a otras donde se tiene en cuenta el material con el que está confeccionada la propia bóveda. Entre estas formulaciones del siglo xviı y posteriores, Oliveira et al. (18), Manjón (12) y Martín-Caro (15) citan las que se indican a continuación, si bien se debe destacar que la fecha que Figura entre paréntesis es la de la publicación de cada una de ellas, aunque estaban siendo utilizadas con anterioridad, para una vez contrastados sus resultados, hacerlas públicas.

Algunas de estas fórmulas están expresadas en función de la Luz $(L)$, y otras en función del radio $(r)$, por lo que utilizando la expresión que relaciona ambos, indicada con anterioridad, se han transformando aquellas expresiones que ofrecían esa posibilidad, en función del rebajamiento, quedando homogeneizadas de la siguiente manera:

\section{Gautier (1714 s. XVIII)}

$$
\begin{array}{cl}
e=\frac{L}{18} \text { Para } L<10 \text { m y piedra dura en arcos de medio punto. } \\
e=0,32+0,067 L \quad \begin{array}{l}
\text { Para piedra blanda en arcos de medio } \\
\text { punto. }
\end{array}
\end{array}
$$

\section{Perronet (1777 S. XVIII) (19)}

\section{Gauthey (1809 S. XIX) (20)}

$e=0,33+0,021 \cdot L$ para $2<L<16 \mathrm{~m}$ Para arco de medio punto. 
$e=\frac{L}{24}$ Para $16<L<32 \mathrm{~m}$ Para arco de medio punto. $e=0,67+0,021 \cdot L \quad$ Para $L>32 \mathrm{~m}$ en arco de medio punto.

\section{Sganzin (1809 S. XIX)}

$e=0,325+0,3472 L \quad$ Para arco de medio punto.

\section{Déjardin (1845 S. XIX)}

$e=0,30+0,045 L$ Para medio punto.

$e=0,30+0,041 L$ Para circulares rebajados a $1 / 6$.

$e=0,30+0,037 L$ Para circulares rebajados a $1 / 8$.

$e=0,30+0,026 L$ Para circulares rebajados a $1 / 10$.

\section{L'Eveille (1854 s. XIX)}

$e=0,33+0,033 L \quad$ Para arcos de medio punto.

$e=0,33+0,033 \sqrt{L}$ Para arcos rebajados.

\section{Rankine (1862 S. XIX)}

$e=9 \cdot 10^{-3}+\sqrt{8,12 \cdot 10^{-5}+18 \cdot 10^{-2} L}$ Arcos de medio punto

\section{Dupuit (1870 s. XIX)}

$e=0,20 \cdot \sqrt{L}$ Para arcos de medio punto.

$e=0,15 \cdot \sqrt{L}$ Para arcos rebajados.

\section{Croizette-Desnoyers (1885 s. XIX)}

$e=0,15+0,142 \cdot \sqrt{L} \quad$ Para arcos de medio punto

$e=0,15+0,183 \cdot \sqrt{L}$ Para arcos rebajados a $1 / 6$.

$e=0,15+0,206 \cdot \sqrt{L}$ Para $\operatorname{arcos}$ rebajados a $1 / 8$.

\section{Elzeario Boix (s. XIX)}

$$
e=\frac{\sqrt[3]{L}}{3}
$$

FORMULACION DE Perronet (1777)

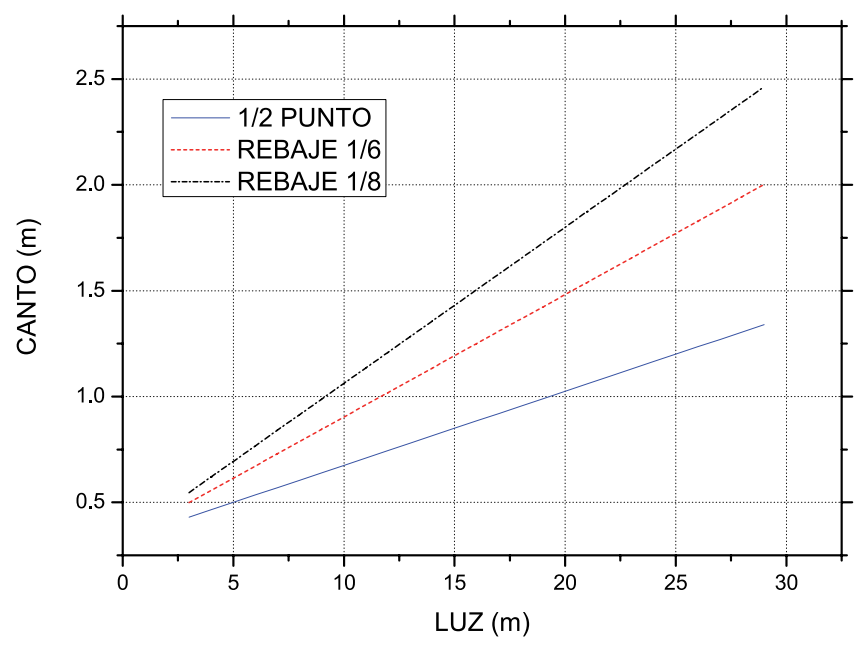

Figura 4. Gráfica comparativa de la fórmula de Perronet para distintos tipos de arcos.

\section{Luis Gaztelu. M de Echandía (s. XIX)}

$$
e=1,35+0,75 \cdot \sqrt{L}-1,40 \cdot \sqrt[3]{L}
$$

Séjourné (1914 S. Xx)

$$
e=0,15 \cdot(1+\sqrt{L}) \cdot v
$$

En la que: $v=\frac{4}{3} \cdot\left(1-\mu+\mu^{2}\right)$

$\mu=$ rebajamiento de los $\operatorname{arcos}(f / L)$.

Con lo que dando valores al rebajamiento se tendrá:

$$
\begin{aligned}
& v=1,04 \text { Para un rebajamiento de } 1 / 3 . \\
& v=1,08 \text { Para un rebajamiento de } 1 / 4 . \\
& v=1,12 \text { Para un rebajamiento de } 1 / 5 . \\
& v=1,15 \text { Para un rebajamiento de } 1 / 6 . \\
& v=1,19 \text { Para un rebajamiento de } 1 / 8 .
\end{aligned}
$$

En cualquier caso se aprecia que el coeficiente $v$ es directamente proporcional al espesor y también es creciente conforme aumenta el rebajamiento.

Podemos destacar que hasta el siglo xviII apenas se efectuaba distinción entre un arco de medio punto y uno rebajado, lo cual pudiera ser un indicador de que la tendencia habitual era la utilización del primero. Solamente Perronet hace esa distinción, haciendo crecer el espesor conforme aumentaba el rebajamiento y en cualquier caso también conforme aumentaba la luz (Figura 4). En el siglo XIx Déjardin propone utilizar menos canto para el arco escarzano que para el arco de medio punto a igualdad de luz, como puede apreciarse en la Figura 5. Posteriormente Dupuit mantiene un criterio similar.

Sin embargo, ya a finales de ese siglo xIx donde el uso del escarzano está más generalizado, quienes formulan expresiones distinguiendo el tipo de arco lo hacen de forma contraria, dando mayor espesor de bóveda a los rebajados que al de medio punto a igualdad de luz. Un ejemplo de ello son las formulaciones de Croizette-Desnoyers primero, y de Séjourné después (Figura 6).

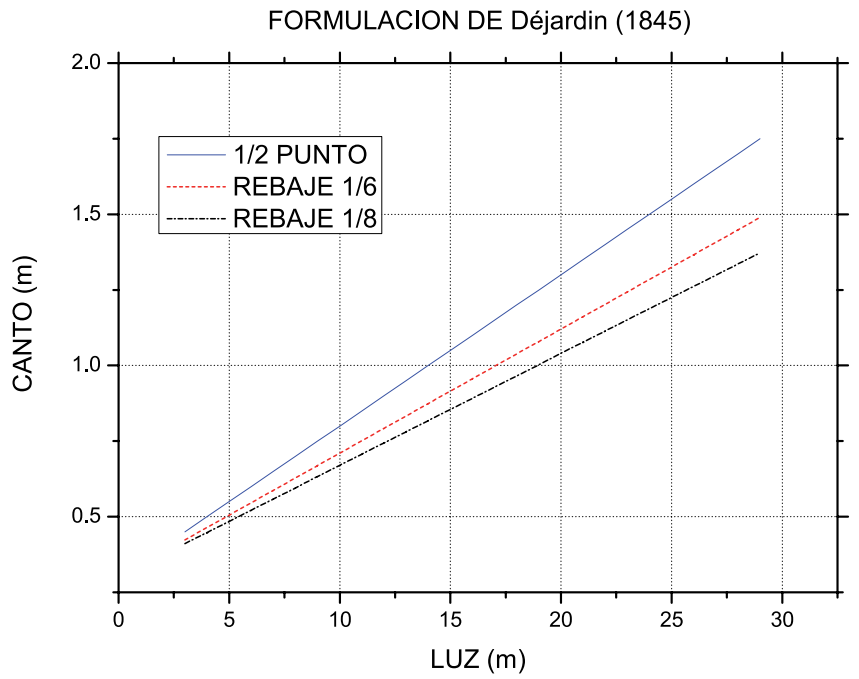

Figura 5. Gráfica comparativa de la fórmula de Déjardin para distintos tipos de arcos. 

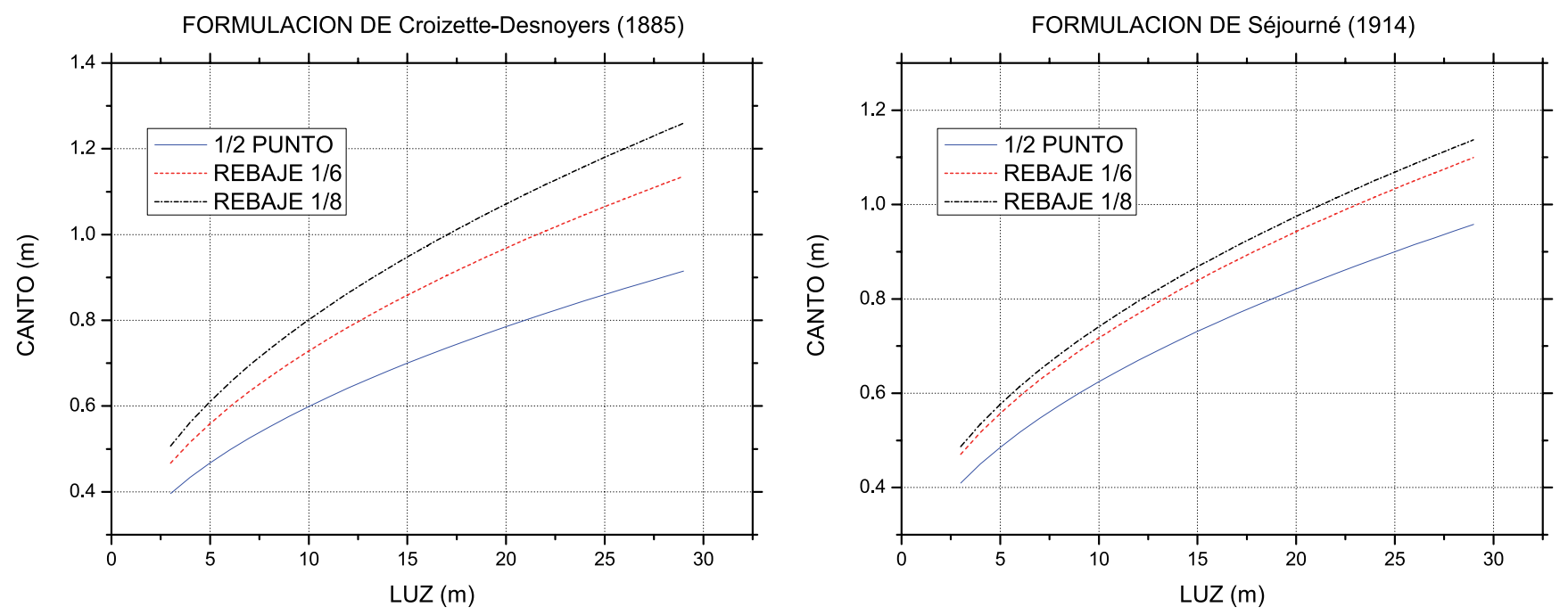

Figura 6. Gráfica comparativa de las fórmulas de Croizette-Desnoyers y de Séjourné para distintos tipos de arcos.
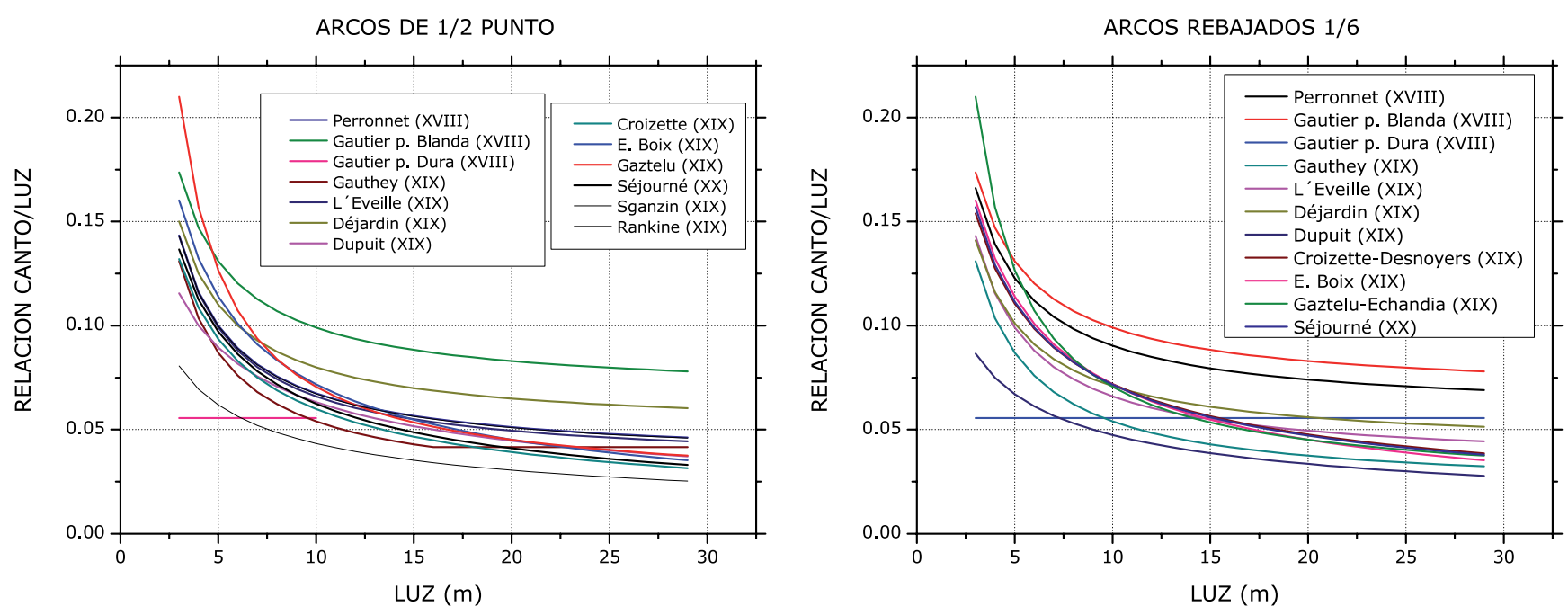

Figura 7. Gráfica comparativa de la variación de la relación canto/luz con la luz para arcos de medio punto y escarzanos con rebajamiento a $1 / 6$.

Un aspecto común a todas las formulas expuestas es que conforme aumenta la luz disminuye la relación espesor de rosca luz $\left(e_{r} / L\right)$, debido a la no linealidad de ésta última (Figura 7), como también aprecian Martínez et al. (21). La variación es más apreciable cuanta más pequeña es la luz y se da tanto en arcos de medio punto como en rebajados.

\section{ANÁLISIS DE LA VARIACIÓN DE LA CARGA DE ROTURA}

Los trabajos desarrollados por Martín-Caro (15), González Parejo (9) y Ramos Casquero (17) han puesto de manifiesto que la posición de la carga, el relleno rígido y el rebajamiento influyen en el valor de la carga de rotura en la bóveda. González Parejo (9) estudió, para este tipo de estructuras de fábrica, la variación de la carga máxima admisible en función de la posición. Para el caso de bóvedas de medio punto realizó su análisis partiendo desde una posición inicial de $0,15 \mathrm{~L}$ tomando diferentes alturas de relleno a partir de $0,5 f$, dentro de un análisis paramétrico más amplio.

En el presente trabajo se analizan distintas directrices de arco, desde el ángulo $\theta$ de $23^{\circ}(1 / 20)$, hasta llegar a $180^{\circ}$
(1/2), pasando por $38^{\circ}(1 / 12), 56^{\circ}(1 / 8), 74^{\circ}(1 / 6), 87^{\circ}(1 / 5)$, $105^{\circ}(1 / 4), 135^{\circ}(1 / 3)$ y $155^{\circ}(1 / 2,5)$. De esta manera el estudio no se ha ceñido a la bóveda de medio punto y, además, se ha tenido en cuenta la posibilidad de que exista o no relleno rígido, tomando como altura del mismo los 2/3 de la flecha. Esta hipótesis de altura de relleno proporciona la configuración más estable de forma coherente a lo señalado por Ramos Casquero (17), como se ha mencionado anteriormente. De esta forma se amplía el rango y las variables establecidas en el trabajo de González Parejo (9).

Para realizar los cálculos se ha recurrido al programa Archie-M (v. 2.3.1), elaborado por Bill Harvey, Fraser Smith y Zoltàn Juhàsz para OBVIS Ltd. (Exeter-United Kingdom) en 2010, con el apoyo del Science and Engineering Research Council (EPSRC), y en colaboración con Bill Harvey Associates Ltd. Dicho programa realiza el estudio en el plano, y está diseñado para el análisis de puentes de mampostería, basado en el modelo de la línea de presiones. El software precisa pocos datos (geometría, distribución de cargas y peso específico), representando el nivel de colapso más común en este tipo de estructuras, que es la formación de mecanismos cinemáticos. 
Se ha partido de una serie de hipótesis básicas dentro de este método de análisis límite, teniendo en cuenta que lo que se pretende es comparar la variación en el valor de la carga de rotura para diferentes rebajamientos de la bóveda. No obstante para el establecimiento de las conclusiones se realiza un estudio comparativo que no se ciñe estrictamente al valor cuantitativo de ésta sino a su evolución e influencia en términos cualitativos, por lo que el valor de algunas de las propiedades iniciales del modelo no influye en el objetivo del trabajo.

De esta forma, todos los análisis se han llevado a cabo para la misma tipología de bóveda mono-arco con una luz de $8 \mathrm{~m}$, y un espesor de bóveda de $0,60 \mathrm{~m}$. El peso específico adoptado para la fábrica ha sido $24 \mathrm{kN} / \mathrm{m}^{3}$, de $20 \mathrm{kN} / \mathrm{m}^{3}$ para el relleno rígido y $18 \mathrm{kN} / \mathrm{m}^{3}$ para el relleno granular. El programa utilizado no considera posibles los desplazamientos entre elementos, y la resistencia a tracción de la fábrica se ha considerado nula. La resistencia a compresión propuesta es de $30 \mathrm{MPa}$, pues como demuestra González Parejo (9) al efectuar el análisis de la carga de rotura variando la resistencia de la fábrica, a partir de un valor de comprendido entre 7,5 MPa y $10 \mathrm{MPa}$ el aumento de esta resistencia tiene poca o nula repercusión en la carga de rotura de la bóveda, por ser prácticamente constante. Por lo tanto, su influencia a partir de esos valores sería despreciable. Para el relleno rígido se ha mantenido el mismo criterio que el adoptado por González Parejo (9) en el análisis estructural efectuado sobre diferentes bóvedas en su Tesis, basado en estudios desarrollados en el Departamento de Mecánica de los Medios Continuos y Teoría de Estructuras de la Escuela de Caminos de la Universidad Politécnica de Madrid. En este trabajo consideró como hipótesis asimilar las características mecánicas del material del relleno rígido al de las piezas de la fábrica de la bóveda. Esta misma hipótesis de partida fue adoptada por Martín Caro (15) en su Tesis, y coincide con lo expuesto por Espejo y León (22) en su trabajo sobre el comportamiento experimental de los puentes de fábrica ferroviarios de la Riera de Rubí y de Plazaola en Urnietasu, ensayados hasta rotura. Sus valoraciones justificaron la importancia del relleno rígido en la respuesta final, indicando que proporciona cierta continuidad estructural a la bóveda, mejorando la estabilidad.

Con todo ello, se ha ido desplazando la carga a lo largo de la rasante superior de la estructura y aumentando su valor, añadiéndose además la carga permanente correspondiente al peso propio. El reparto de la carga se efectúa a través de conos de eje vertical con semi-ángulo de $45^{\circ}$ para el relleno rígido, de manera similar a los trabajos previamente realizados por Ramos Casquero (17) y Martín Caro (15).

En primer lugar se ha determinado la posición más desfavorable de la carga en cada tipo de bóveda, tanto considerando la existencia de relleno rígido en los $2 / 3$ de la flecha, como sin considerarla. Para ello se ha calculado el valor de la carga de rotura en función de la posición de la carga para distintas fracciones de $L$, desde o hasta $0,5 \mathrm{~L}$.

\subsection{Análisis de la carga de rotura en cada tipo de bóveda en función de la posición de la carga (influencia del relleno rígido)}

Los resultados obtenidos en los cálculos permiten apreciar que la existencia de relleno rígido como elemento colaborante en la resistencia de la bóveda afecta más a la posición pésima de la carga cuanto menos rebajada es la bóveda (Figura 8), pues es en ese tipo de directrices donde la posición del valor mínimo de la carga de rotura se encuentra más distante entre las curvas correspondientes a cada caso (con relleno rígido y sin él). Ello se debe a que dicho relleno rígido puede provocar tanto un cambio en el rebajamiento como una variación de luz eficaz de trabajo.

En las bóvedas de medio punto la modificación en el rebajamiento originado por el relleno rígido es notable, al pasar a una bóveda escarzana con un rebajamiento próximo a 1/5 (Figura 9). Esto hace que se modifique la forma de trabajar de la bóveda, tal como puede advertirse al comparar la forma de las curvas de carga de rotura correspondiente a ambos casos (con y sin relleno rígido) (Figura 8).

A partir del rebajamiento de 1/5 la presencia del relleno rígido no origina un cambio tan sustancial en la tipología: las rebajadas a $1 / 3$ pasarían a tener un rebajamiento próximo a $1 / 6$, mientras una escarzana a $1 / 5$ pasaría a tener un rebajamiento próximo a 1/8 (Figura 9). Por este motivo las diferencias entre las curvas se van haciendo cada vez menos acusadas según aumenta el rebajamiento, atenuándose hasta hacerse similares (Figura 8). También se aprecia dicha atenuación en la concavidad de las curvas, tendiendo en el límite (viga plana) a una curva monótona decreciente.

En aquellas tipologías en las que el relleno rígido tiene una mayor influencia se produce una reducción de la luz efectiva, medida como distancia entre los puntos de corte entre las líneas de presiones y la bóveda (Figura 11); esto provoca que la carga de rotura sea mayor cuando existe dicho relleno rígido que cuando no existe y, por consiguiente, que la resistencia sea mayor (Figura 8).

\subsection{Análisis de la carga de rotura para la posición más desfavorable de la carga (influencia del rebajamiento y del relleno rígido)}

Tal como ya se ha señalado, el trabajo desarrollado por González Parejo (9) puso de manifiesto la influencia del rebajamiento en la variación de la carga de rotura. Sus análisis abarcaron desde la bóveda de medio punto hasta la rebajada a 1/3. En este trabajo se ha completado dicho análisis llegando hasta la bóveda escarzana con rebajamiento de 1/20, e incluyendo la influencia del relleno rígido. Para ello, y como continuación al análisis expuesto en el punto anterior, se ha determinado la carga de rotura para la posición más desfavorable de la misma en cada tipo de bóveda, previamente obtenida para cada uno de ellos, teniendo en cuenta la posibilidad o no de participación del relleno rígido en la respuesta resistente.

Los resultados bajo estas premisas señalan que en el caso de existencia de dicho relleno rígido la carga de rotura que se obtiene va aumentando desde la bóveda de medio punto hasta la bóveda rebajada a 1/3. Este aspecto fue también indicado por González Parejo (9). Al analizar los datos y gráficas obtenidas puede deducirse que la explicación a este fenómeno se encuentra en la reducción de la luz efectiva de la bóveda que se produce al tener en cuenta el relleno rígido en los casos de rebajes inferiores a 1/3 (Figura 11). Esto, unido a que ese rebajamiento era el más habitual en tiempos de Déjardin, hace 

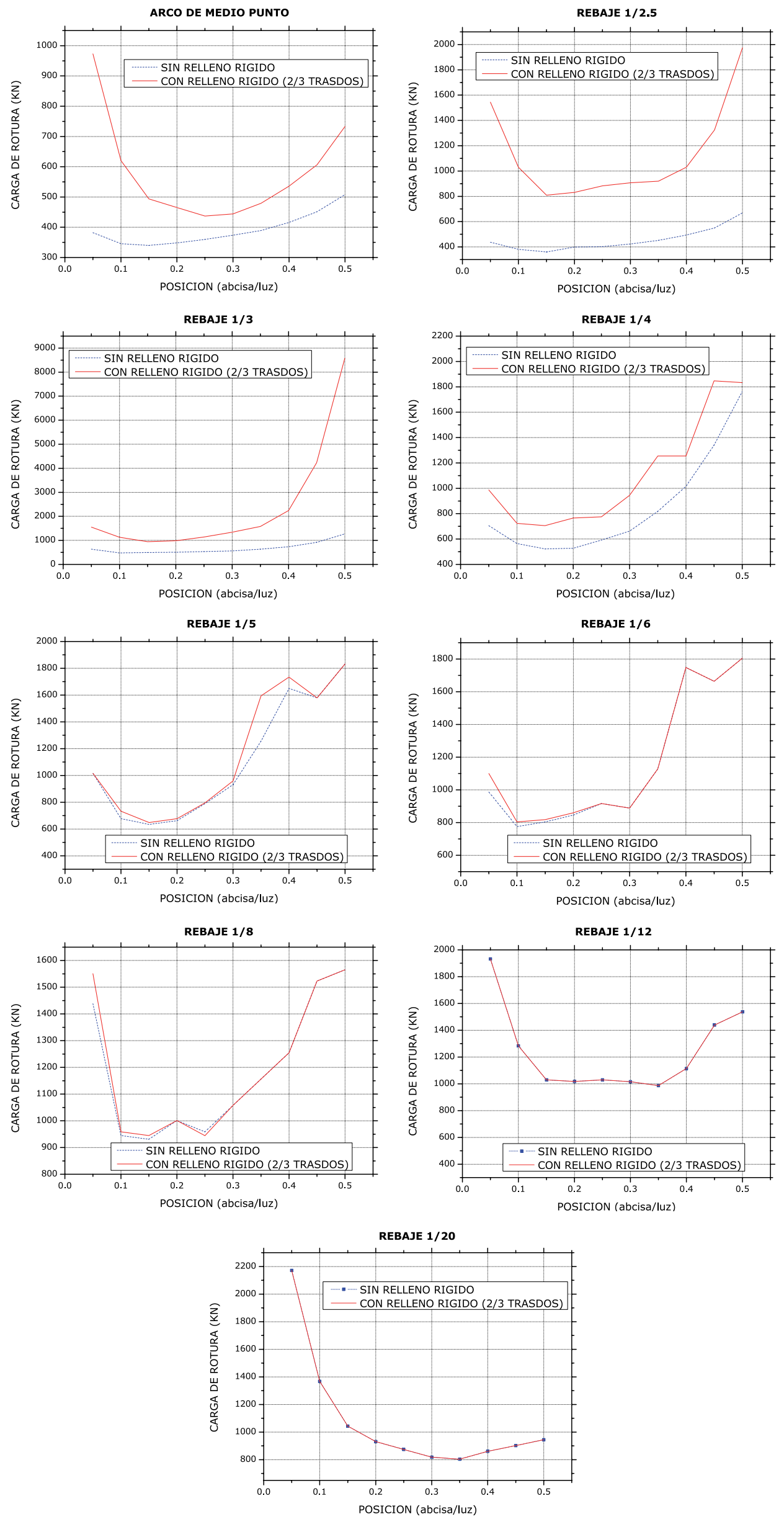

Figura 8. Gráficas de la variación de la carga de rotura con la posición de la carga, con relleno rígido y sin él. 


\section{ARCO DE MEDIO PUNTO}

(REBAJAMIENTO 1/2)

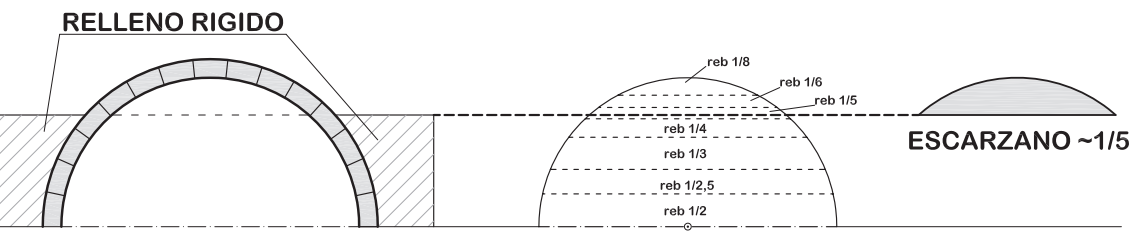

\section{ARCO REBAJADO (REBAJAMIENTO 1/3)} RELLENO RIGIDO
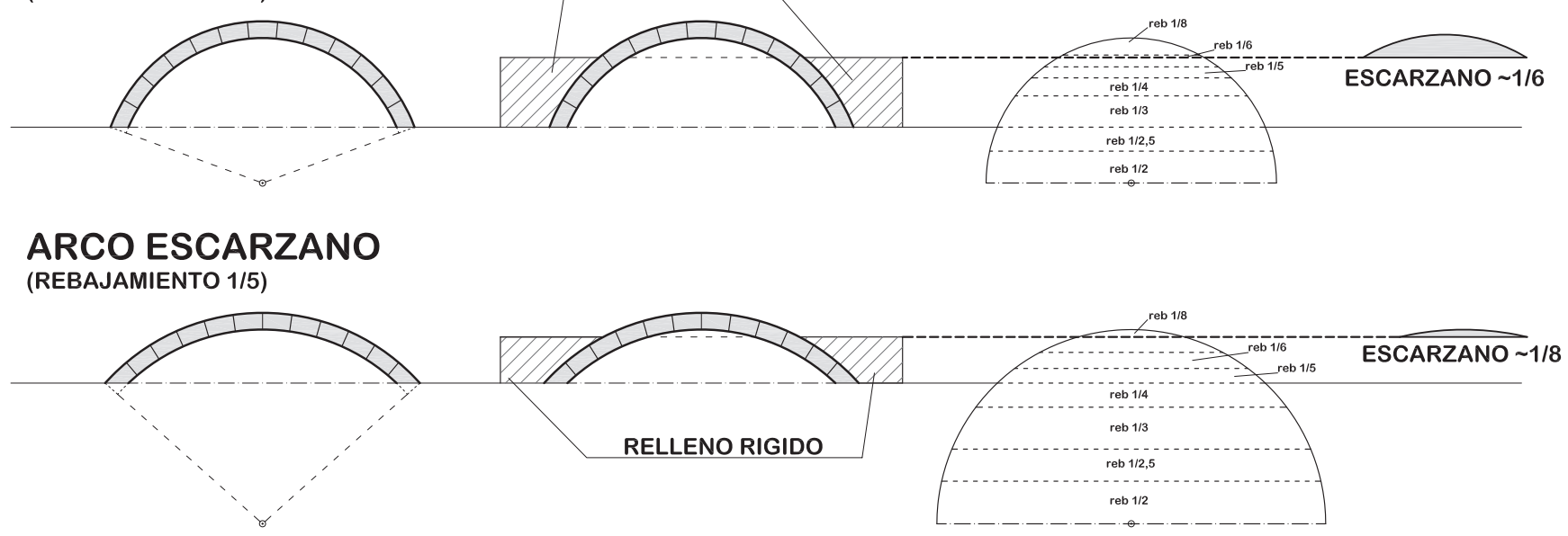

Figura 9. El relleno rígido influye variando el rebajamiento de forma más o menos acusada, en función del tipo de bóveda de que se trate.

que las conclusiones de este autor fueran particularmente certeras.

Al completar el espectro de bóvedas analizadas por González Parejo (9), llegando hasta la escarzana de ángulo de $23^{\circ}$ (rebajamiento 1/20), hemos podido advertir que es precisamente en la de $1 / 3$ cuando se obtiene un máximo en el valor de la carga de rotura. Pero conforme se va «aplanando» la directriz de la bóveda, el valor de la carga vuelve a reducirse hasta llegar a la bóveda escarzana con rebajamiento de 1/5. A partir de este valor se comporta como una bóveda sin relleno rígido, pues, como se ha indicado anteriormente, a partir de ese rebajamiento el relleno rígido tiene poca influencia. La explicación a estas observaciones requiere tener en cuenta dos efectos: por una parte la línea de presiones deja de tocar a puntos del interior de la bóveda con el consiguiente aumento de la luz efectiva (Figura 12), y por otra, se va produciendo un aumento en el radio de curvatura, que a igualdad de luz efectiva es el factor diferenciador.

En el caso de no existir relleno rígido (Figura 10), la carga de rotura es creciente hasta alcanzar un valor máximo en torno al rebajamiento de $1 / 12$, debido a la reducción de luz efectiva. Es a partir de este punto cuando se reduce el valor de la carga de rotura conforme se «aplana» la bóveda, pues dicha luz efectiva va aumentando hasta llegar a su dimensión real (Figura 12).

Además, la diferencia entre la respuesta tensional con y sin relleno (Figura 10) se hace cada vez menor a medida que aumenta el rebajamiento: por un lado, llega un momento en que la luz efectiva es igual a la luz real (Figura 12), sin que influya en mayor medida este efecto; por otro, a medida que aumenta el radio de curvatura el comportamiento se hace más homogéneo para ambos casos. Es decir, que cuanto

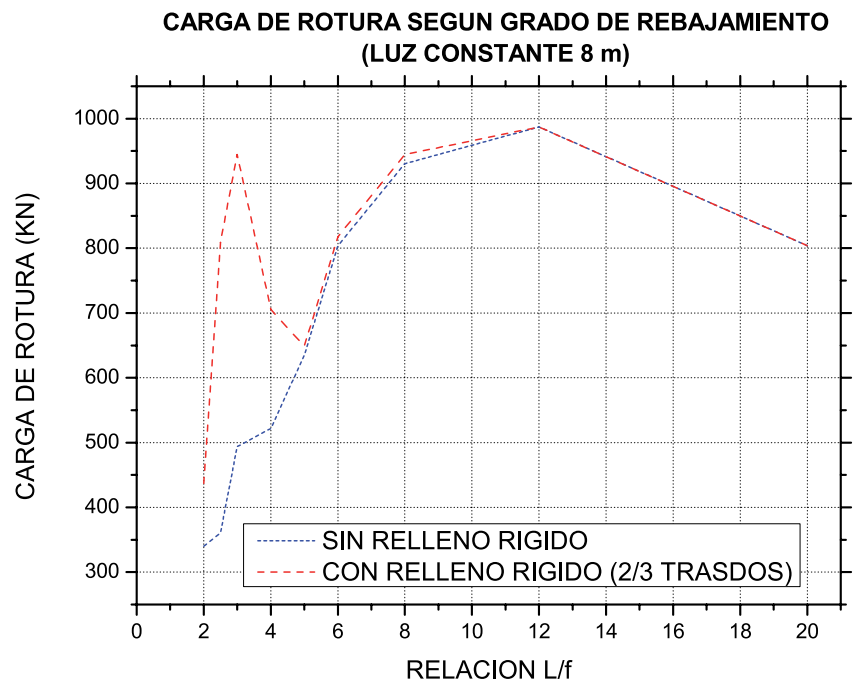

Figura 10. Variación de la carga de rotura en función del rebajamiento de la bóveda con y sin relleno, para luz constante.

más rebajada es la bóveda, la influencia del relleno rígido es menor, pues por una parte la línea de presiones no produce reducción de luz efectiva, y por otra, el cambio que produce el relleno en la forma de trabajo de la bóveda es menos apreciable.

\section{CONCLUSIONES}

De los estudios previos llevados a cabo sobre diferentes puentes se ha podido comprobar que el tipo de directriz circular única más utilizada en los puentes ha sido el arco de medio punto, así como que la disminución del valor del rebajamiento ha ido en aumento con el devenir del tiempo. 

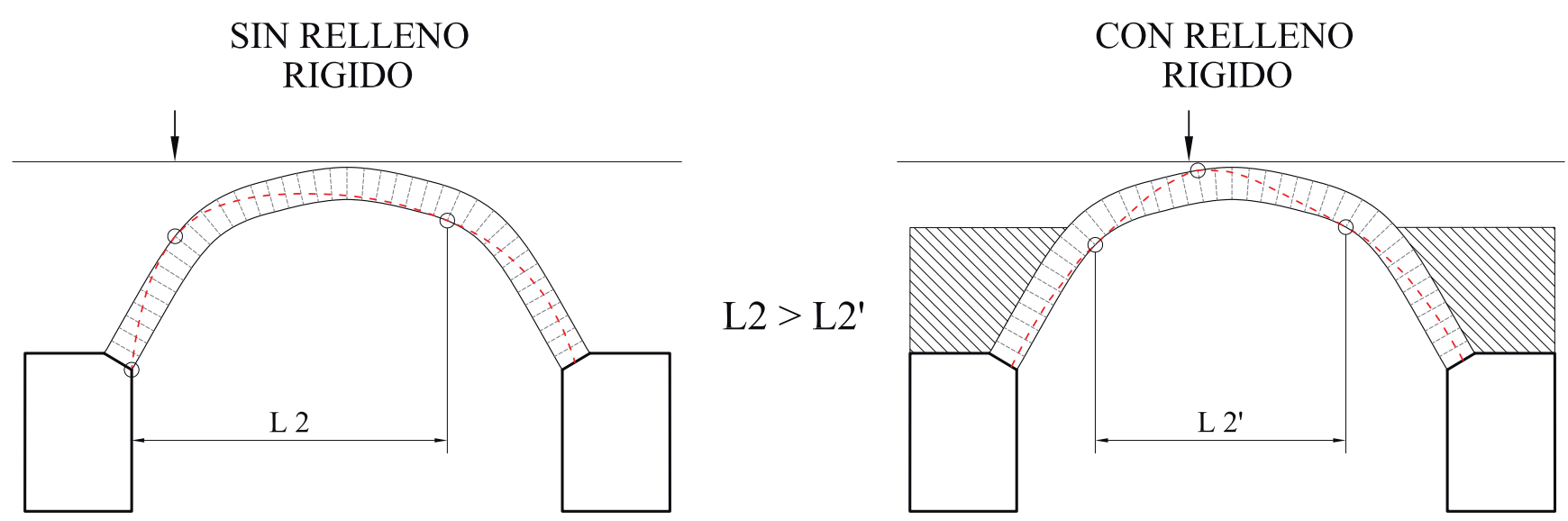

Figura 11. Variación de las luces eficaces sin relleno rígido (izquierda) y con él (derecha), para la posición más desfavorable de carga en cada caso (rebajamiento $<1 / 3$ ).
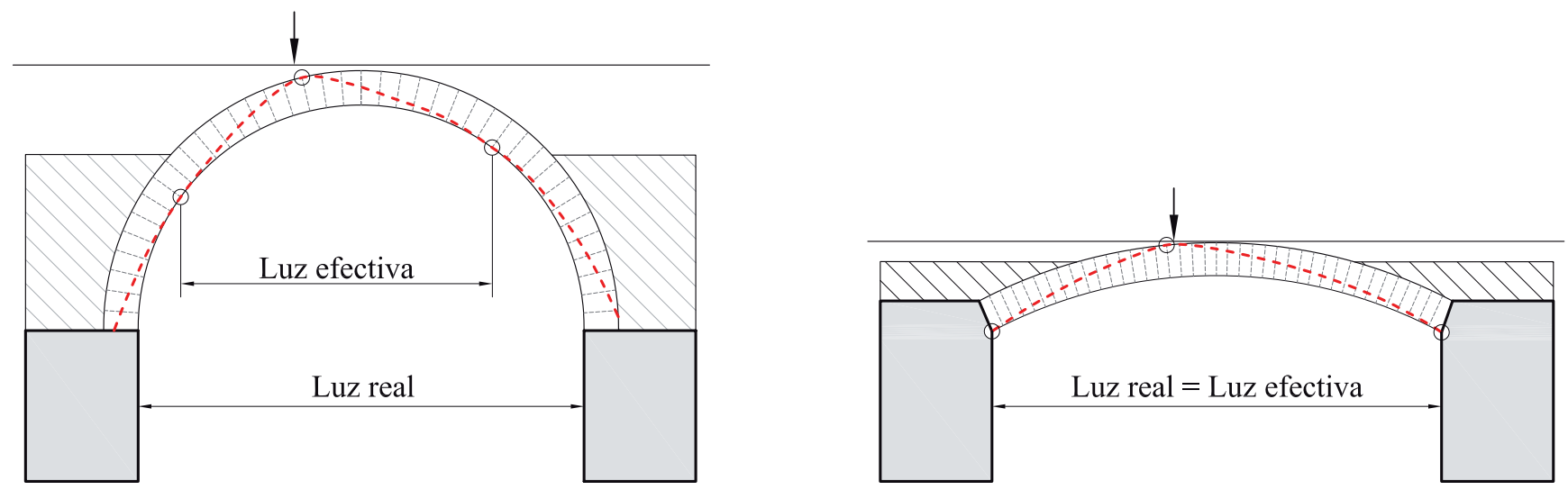

Figura 12. Los resultados de cálculo permiten comparar la línea de presiones para una bóveda de medio punto (izquierda) y uno con rebajamiento $1 / 6$ (derecha) obtenida al tener en cuenta el efecto del relleno rígido. A partir de cierto rebaje la luz efectiva iguala a la real sin que ésta pueda incrementarse más.

Avalan estas afirmaciones los estudios llevados a cabo tanto en la cuenca del río Arlanza por Manjón (12), como en el análisis efectuado por González Parejo (9) en los puentes de la provincia de Cáceres, así como los datos obtenidos en un estudio que nos encontramos realizando en puentes del río Carrión (Palencia). Los cálculos analizados en este artículo han permitido constatar la evolución de la carga de rotura en función de la posición para distintos rebajamientos con y sin presencia de relleno rígido. Los resultados obtenidos han permitido corroborar las estimaciones cualitativas ya señaladas por Espejo y León (22) y por Ramos Casquero (17) respecto a la influencia del relleno rígido en el comportamiento estructural de los puentes con bóveda de piedra. Así, no sólo debe de tenerse en cuenta la geometría del mismo, es decir, la forma exterior del puente, sino también otros factores. Por ejemplo, la presencia de una capa de relleno rígido modifica sustancialmente la respuesta estructural. Este aspecto es especialmente importante cuanto menos rebajada es la bóveda, mientras que las diferencias tienden a diluirse a medida que aumenta el rebajamiento.

Igualmente se ha comprobado que con la presencia de relleno rígido la carga de rotura aumenta desde la bóveda de medio punto hasta el que tiene un rebajamiento de $1 / 3$, como ya puso de manifiesto González Parejo (9). Con los nuevos cálculos aportados puede añadirse que a partir de este rebajamiento el valor de la carga de rotura se reduce hasta que deja de tener tanta influencia el relleno rígido, comportándose entonces como una bóveda sin relleno rígido. Este aspecto no se veía reflejado en los tratados y formulaciones enunciadas en los siglos xviII y xix. Una combinación de efectos permite explicar este comportamiento: por un lado, cuanto menor es el rebajamiento de la bóveda, mayor es el cambio de funcionamiento estructural que supone la existencia de relleno rígido; por otro, la reducción de luz efectiva que produce la presencia de dicho relleno rígido alcanza un valor mínimo con el rebajamiento de 1/3, para luego comenzar a aumentar.

Por último, la diferencia entre la respuesta tensional con y sin relleno (Figura 10) se hace cada vez menor a medida que aumenta el rebajamiento: por un lado, a partir de un cierto rebaje la luz efectiva es igual a la luz real (Figura 12), sin que influya en mayor medida este efecto; por otro, a medida que aumenta el radio de curvatura el comportamiento se hace más homogéneo para ambos casos. Es decir, que cuanto más rebajada es la bóveda, la influencia del relleno rígido es menor, pues, por una parte la línea, de presiones no produce reducción de luz efectiva, y por otra, el cambio que produce el relleno en la forma de trabajo de la bóveda es menos apreciable. 


\section{REFERENCIAS}

(1) Huerta Fernández, S. (2004). Arcos, bóvedas y cúpulas. Geometría y equilibrio en el cálculo tradicional de estructuras de fábrica. Madrid: Instituto Juan de Herrera, Escuela Técnica Superior de Arquitectura.

(2) Arenas de Pablo, J. J. (2002). Caminos en el aire. Colección ciencias, humanidades e ingeniería, vol. 1. Madrid: Colegio de Ingenieros de Caminos, Canales y Puertos.

(3) Fernández Casado, C. (2009). Historia del puente en España: puentes romanos. Madrid: Colegio de Ingenieros de Caminos, Canales y Puertos.

(4) Serrano-López, R., Mínguez-Algarra, J., Cambronero-Barrientos, F., Saldaña-Arce, D. (2014). La adaptación de la forma a las cargas en el arte estructural: desde las pilas y el arco, a la torre. Informes de la Construcción, 66(534): e021, doi: http://dx.doi.org/10.3989/ic.13.005.

(5) Bauder, E. (2006). ¿Son seres humanos los puentes de fábrica? Are masonry bridges human beings? Informes de la Construcción, 58 (501): 33-46, doi: http://dx.doi.org/10.3989/ic.2006.v58.i501.397.

(6) Grupo de trabajo «Puentes de Fábrica» del Comité de Puentes de la Asociación Técnica de Carreteras (ATC-AIPCR). (2009). Criterios y técnicas de ensanche de tableros en puentes de fábrica. Madrid: Asociación Técnica de Carreteras.

(7) Grupo de trabajo «Puentes de Fábrica» del Comité de Puentes de la Asociación Técnica de Carreteras (ATC-AIPCR). (2003). Ensillado de bóvedas. Revista RUTAS (96), mayo-junio, Separata.

(8) Grupo de trabajo «Puentes de Fábrica» del Comité de Puentes de la Asociación Técnica de Carreteras (ATC-AIPCR). (2014). Criterios de intervención en puentes de fábrica. Madrid: Asociación Técnica de Carreteras.

(9) González Parejo, J. M. (2014). Puentes de fábrica, romanos y medievales en la provincia de Cáceres. Tesis doctoral dirigida por Siegrist Fernández, C., y Rodado López, J. Madrid: Universidad Politécnica de Madrid, http://oa.upm.es/30316/.

(10) Ramos Casquero, A., León González, J. (2013). Clasificación morfológica de los rellenos en el trasdós de bóvedas de fábrica. Informes de la Construcción, 65(532): 471-480, doi: http://doi.org/10.3989/ic.12.062.

(11) Grupo de trabajo «Puentes de Fábrica» del Comité de Puentes de la Asociación Técnica de Carreteras (ATC-AIPCR). (1999). Primer glosario de términos empleados en puentes de fábrica. Revista RUTAS (70), enero-febrero, Separata.

(12) Manjón Miguel, J. L. (2006). Los puentes de fábrica del río Arlanza. Análisis de su construcción y propuestas de datación. Tesis dirigida por Martínez Martínez, J. A (Tesis doctoral no publicada). Universidad de Burgos.

(13) Aramburu-Zabala Higuera, M. A. (1992). La arquitectura de puentes en Castilla y León 1575-1650, pp. 12-86. Valladolid: Junta de Castilla y León.

(14) Marías y Bustamante, F. (1983). Un tratado inédito de arquitectura de hacia 1550. Madrid: Biblioteca Nacional.

(15) Martín-Caro Álamo, J. A. (2001). Análisis estructural de puentes arco de fábrica. Criterios de comprobación. Tesis dirigida por León González, J. Universidad Politécnica de Madrid.

(16) Huerta Fernández, S. (2000). Estática y geometría: el proyecto de puentes de fábrica en los siglos xv al xviI. Actas del Tercer Congreso Nacional de Historia de la Construcción. Sevilla: Sociedad Española de Historia de la Construcción.

(17) Ramos Casquero, A. (2015). Caracterización estructural de los rellenos situados en el trasdós de bóvedas de edificios históricos. Tesis dirigida por León González, F. J. Madrid: Universidad Politécnica de Madrid, http://oa.upm.es/38758/.

(18) Oliveira, D. V., Lourenço, P. B., Lemos, C. (2010). Geometric issues and ultimate load capacity of masonry arch bridges from the northwest Iberian Peninsula. Engineering Structures, 32(12): 3955-3965, doi: http://doi.org/10.1016/j.engstruct.2010.09.006.

(19) Perronet, J. R. (1746). Memoire sur lépasseur que doivent avoir les voûtes des ponts avec des tables et expèriences. Paris: École des Ponts et Chaussées.

(20) Gauthey, F. M. (1845). Traité de la construction des ponts. Liége: Chez Leduc, Librarie.

(21) Martínez, J. L., Martín-Caro, J. A., León, J. (2003). Monografias sobre el análisis estructural de construcciones históricas de fábrica. Evaluación estructural de puentes arco de fábrica. Departamento de Mecánica de Medios continuos y teoría de estructuras. Madrid: Universidad Politécnica de Madrid.

(22) Espejo, J. (2007). Estudio del comportamiento experimental de los puentes de fábrica ensayados hasta rotura (Puente ferroviario de la Riera de Rubí y Puente sobre el ferrocarril de Plazaola en Urnietasu). Trabajo de investigación tutelado por León, M. Madrid: Universidad Politécnica de Madrid. 